\title{
Implementing Delaunay Triangles and Bezier Curves to Identify Suitable Business Locations in the Presence of Obstacles
}

\author{
Tejas Pattabhi \\ PESIT South Campus, Bangalore, India \\ tejas.pattabhi@gmail.com \\ Arti Arya \\ PESIT South Campus, Bangalore, India \\ artiarya@pes.edu \\ Pradyumna N \\ PESIT South Campus, Bangalore, India \\ pradyumna.nagendra@gmail.com \\ Swati Singh \\ PESIT South Campus, Bangalore, India \\ swati04singh@gmail.com \\ Sukanya D \\ PESIT South Campus, Bangalore, India \\ indhu19dhansingh@gmail.com
}

\begin{abstract}
Data mining plays an important role in collecting information to make businesses more competitive in present business world. It is seen that the location of any business outlet is a major factor of its success. Establishing different business enterprises include a detail study of localities, people's income status living in those areas, and many other non-spatial factors. This paper is one such idea to suggest those locations for entrepreneurs, based on which they can decide on the where they can setup their business outlet. The proposed algorithm makes use of Delaunay triangulation for capturing spatial proximity and Bezier curves are used to model obstacles. The algorithm is implemented as Web application, which accepts the name of a place and collects data, form clusters and show the feasible locations of the service specified, considering the geographic irregularities and man-made obstructions. In this algorithm, spatial and non-spatial data related to a location are collected and the spatial clustering algorithm is in itiated which works based on the obtained data. Clusters are formed based on the unique characteristics of each location. The experimental results are conducted on many different locations of India and in this paper results are shown for three places namely, Mysuru, Patna and Mumbai. The results have shown expected and exciting results.
\end{abstract}

Index Terms - Bezier Curves, Computational Geometry, Delaunay Triangulation, Spatial Data Attributes, Non-Spatial Data Attributes

\section{Introduction}

In today's rapidly changing, highly competitive business world to make maximum profit, data mining plays an important role. Profit made by any business sector depends to a very large extent on its location. Selecting the location for any business enterprise depends on a number of spatial and non-spatial attributes. Relevant data are paramount to an application's capability to provide accurate and actionable information. Various data mining techniques can be useful in providing the solution.

Considering the business point of view, it is seen that the location of any business outlet is one of the essential and critical factors of its success. Any outlet put up in an inappropriate location may lead to financial and economic losses. Analyzing the need of any service at a location is very important. In such scenarios, this algorithm's application can be a guide. It locates the areas where an essential business sector can be established based on spatial and non-spatial attributes. 
This in turn is profitable to the entrepreneur and can earn a good profit out of his service.

The scope of application is in various fields of business. An entrepreneur can select the places most suitable for his/her business without actually visiting the places. A person using this application can narrow down his search to a limited number of places.

Initially, during the setup phase of a business, an entrepreneur can save his time in deciding upon a location. A person new to a place can rely on this application to find locations that are highly profitable for his business. It is also applicable to established enterprises in order to relocate or open more number of outlets within a city. For example, a school, established in one area, can open its branches further in various localities of the same city. This application helps in business widening also.

Many marketing firms, business retail outlets, services exist in the market that is not successfully established. Though, there is a need for those products and services, it is not reaching the people demanding for it. This is mainly due to the inappropriate location of those business outlets. This can be avoided if these outlets are in the locations where there is a demand. This problem can be addressed by locating the places of demand. If a solution is found, it is helpful to the businessmen, for earning good profits.

This application that takes in the name of a particular city that the user specifies as input and gives the map marked with the best suitable places as the output. For this application, the spatial and non-spatial data of the city specified, is collected and clustered those places which the user can further survey and setup his business. Before continuing further, a few basic concepts used for developing the proposed algorithm:

\subsection{Spatial Clustering}

Spatial clustering [14] is the technique which groups the spatial objects into groups and is an important component of spatial data mining. In spatial clustering the intra-cluster distance between the objects must be less and inter-cluster distance must be more so as to have well defined clusters.

\subsection{Delaunay Triangles}

Delaunay triangle $[6,15]$ is a geometric structure from computational geometry, which is used for capturing the spatial proximity between the objects as shown in figure 1. For a set of points in a plane, Delaunay Structure is a triangulation such that no point or object is inside the circumcircle of any triangle. It maximizes the minimu $m$ angle of all the triangles in the triangulation. If a set of points is on the same line then Delaunay Triangulation does not exist for such points. If more than three points lie on the same circle then Delaunay Triangulation for such points is not unique. It is highly useful for capturing the spatial proximity between two points.

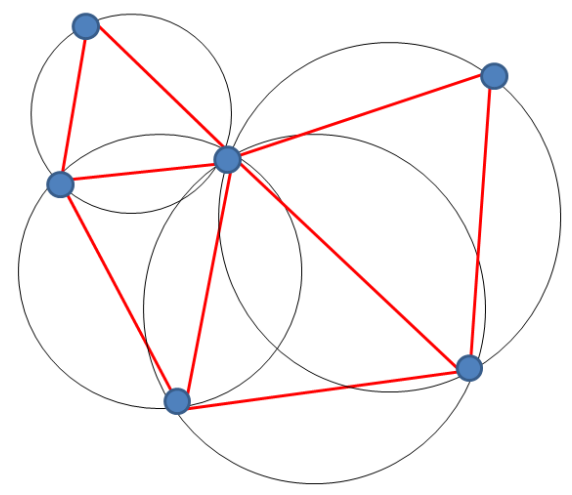

Fig. 1: A Delaunay triangulation

\subsection{Properties of Delaunay Triangulation}

Delaunay structures $[6,7]$ is said to be a collection of edges such that for each edge there is a circle which contains only the end points of the edges and not any other points. A circle, which is circumscribing any triangle in the Delaunay Triangulation, contains only those points that are the vertices of that triangle. The pair of points in Delaunay Triangles that are closest to each other are neighbors. Figure 2 and figure 3 depict when Delaunay condition is not met and when the condition is satisfied.

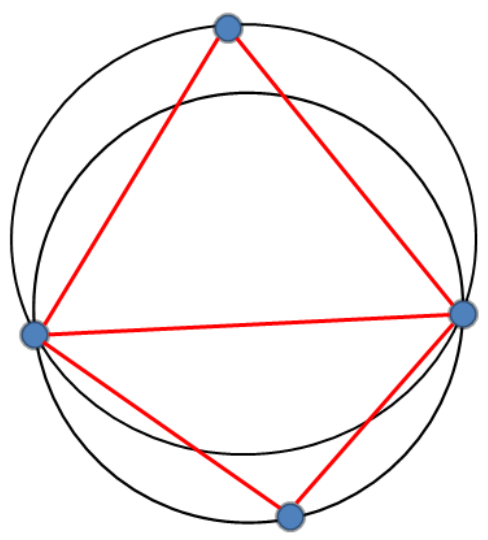

Fig. 2: Delaunay Condition not met

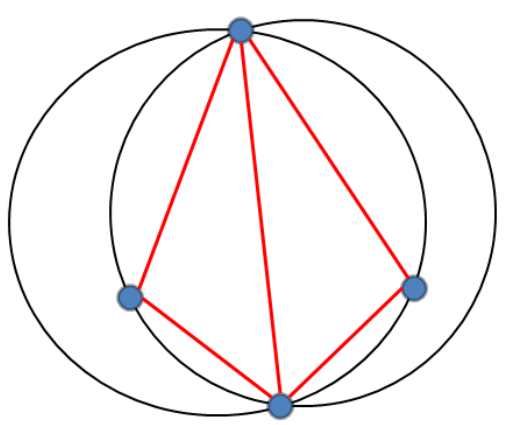

Fig. 3: Flipping the common edge produces Delaunay condition 


\subsection{Bezier Curves}

A set of points in plane called Control points define the Bezier Curve [8]. In figure 4, p $1 \&$ p5 are end points and $\mathrm{p} 2, \mathrm{p} 3 \& \mathrm{p} 4$ are control points. The curve passes through the end points and rest of the points control the shape of the curve i.e. the curve passes through the first and last control points and is pulled towards the intermediate control points [9]. A Bezier Curve of degree $n$ is defined using $n+1$ points.

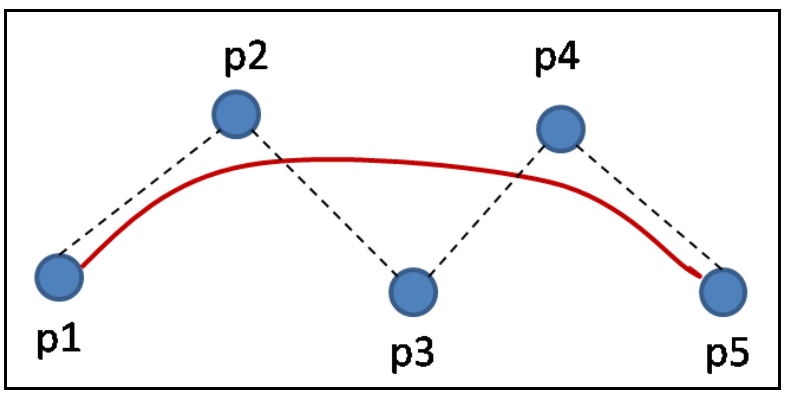

Fig. 4: Bezier curve

A Bezier Curve of degree $d$ is given as [9]

$$
\mathrm{C}(\mathrm{t})=\sum_{i=0}^{d} P_{i} \mathrm{~B}_{i}^{d}(t)
$$

where, $\mathrm{B}_{i}^{d}(\mathrm{t})$ is defined as follows:

$$
\mathrm{B}_{i}^{d}(\mathrm{t})={ }^{d} \mathrm{C}_{i} \mathrm{t}^{i}(1-\mathrm{t})^{d-i}, t \in[0,1] \text { for } i=0, \ldots, \mathrm{d}
$$

The rest of the paper is organized as follows. Section II describes about the literature survey conducted for the proposed work and the limitations of various existing algorith ms. Section III highlights the proposed algorithm for the purpose of spatial clustering of the most appropriate business locations. Section IV describes the experimental results obtained as a result of proposed algorithm. Section V states conclusion and scope of future work.

\section{Related Work}

Zhi-Qiang Liu [4] has implemented FCM (Fuzzy Cognitive Maps) to represent qualitative and quantitative data. Using Contextual fuzzy cognitive maps or CFCM's we can generate using real census data, human expert Knowledge and quantitative data in the form of maps in a GIS. The goal of this system is to use objects and their relationships, either supplied by census data or generated by the GIS and to map them as layers in the GIS. Using fuzzy membership functions we can construct CFCM's for decision support.

It is possible to generate CFCM's from data within geographic information systems (GIS') and human expert reports. For example, if we wish to locate a good location for a "public school" within a new region, not previously known to the GIS but within the boundaries of the human data references, then we would need to query the CFCM, representing the result as a topological region in the GIS. The object is located in the CFCM and its relationships to other objects are represented by bounding circles using the definitions in the GIS and fuzzy membership functions. For example, there may exist relationships between physical objects [i.e., near ("hotel," "main road")], whereas an example of a relationship involving a physical object and a conceptual object might be, contained ("hotel," "typeA_suburb"), where "typeA_suburb" is the conceptual object and the fact that this instance of "hotel" is in a "typeA_suburb" is found from census data. Relationships that involve measurements of concepts such as near, far, decreased, and chance-of are fuzzy. The CFCM's are generated using the definitions, mathematical framework, and generation schemes. For example, if the boundary between near and medium is one third of the resolution at a particular context level, then at the same context level, a boundary one third of the size of the area with its center at the object's location can be used to represent near with respect to another object. The area of greatest interest to an expert for decision support purposes would be the intersection of all of these bounding circles. This, therefore, indicates that this intersection forms: The best possible area for building a "public school."

CFCM now offers the human expert the data necessary to relocate a previously unprofitable positioned landmark to a more profitable position. This analogy can be extended, with the appropriate data sets, to relocating or adjusting river paths, townships, and other landmarks based on the causality constraints between concepts/landmarks contained within CFCM. CFCM allows the human expert to see data within a GIS in ways that was not easily done by other means. Decision support on a greater scale is now possible without having to sift through volumes of otherwise unconnected data. Therefore, CFCM represents a powerful and promising mechanis $m$ for generalizing this data by making the connections among the data assisting human experts in their decision-making process.

Xiang Guo et al.,[2] use Geographic Information Systems (GIS), spatial analysis functions and comprehensive fuzzy sets to evaluate and analyze the ecological suitability of the olive trees in Sichuan, China. The over-years' experience of olive planting and amelioration(enriched) data has also been used as an aid to this analys is taking consideration of climate, soil, and topography which are closely related to the olive growing habit. The results representing the actual distribution of olive while providing alternative adaptive regions for olive growing, thus we are able to offer guidance for the olive cultivation in the region.

Due to its major agricultural importance as the source of olive oil, olive culture has been introduced to the Sichuan Province. Elevation in combination with 
topography leads to vertical differentiation, forming complicated spatial distribution patterns of climate types and soils. GIS and fuzzy comprehensive judgment are employed to analyze and evaluate the ecological suitability for the olive trees. The fuzzy comprehensive judgment is mainly based on fuzzy inference combining qualitative and non-accurate analysis. The standards of evaluation are divided into four ranks, the adaptable, sub-adaptable, minimum-adaptable and non-adaptable. The analytic hierarchy process is used to determine the weight factors in the secondary evaluation model. The judgment matrix is established based on suggestion from experts and the hierarchy structure model. If all the judgment matrices pass the consistency test, the Analytic Hierarchy Process (AHP) levels will be sorted. The membership function is established to evaluate the suitability degree of each basic unit by calculating me mbership degree of each factor to a certain suitability rank. A zoning map of the ecological suitability of Sichuan Olive is obtained through the GIS-based fuzzy comprehensive evaluation. The map obtained through GIS-based fuzzy comprehensive evaluation is able to give very detailed evaluation results.

In this paper, GIS and fuzzy comprehensive evaluation is employed to evaluate the ecological suitability of olive cultivation in Sichuan. In order to ensure an objective and precise evaluation result, analytic hierarchy process is used to obtain the weight of index according to the suggestions of experts while properly selecting the membership function. The results should be adjusted when additional factors such as landslide and debris flow are taken into consideration in practical planning work. The result of this evaluation is able to direct the industry of olive cultivation in Sichuan and helps to generate more economic profits.

H. M. Khodr et al.[3] present a probabilistic methodology; conceived to assist the electric system planning engineers in the selection of the distribution substation locations, taking into account the hourly load changes or the daily load cycle. The hourly load centers, for each of the different hourly load scenarios, are calculated deterministically. This distribution is used to determine the maximum likelihood perimeter of the area where the substation should preferably be located by the planning engineers, taking into account, for example, the availability and the cost of the land lots, which are factors of special relevance in urban areas, as well as other obstacles that may be present in the final selection of the substation site. Results are presented and discussed for the application of the methodology to a real case, assuming three different bivariate probability distributions: the Gaussian distribution, a bivariate version of Freund's exponential distribution, and the Weibull probability distribution.

The location of a substation site depends upon several factors, such as the voltage levels, voltage regulation, substation costs, primary feeders, distribution transformers, and sub-transmission costs, etc. methodologies have been proposed in the literature for the solution of the electric distribution substation. In general, different mathematical programming formulations and techniques have been proposed and applied to the location problem of electrical distribution substations location problem. However, most of these deterministic methodologies are based on the analys is of a constant load level, normally assumed as the maximum load level. This assumption of a constant load level may deviate the solution from a better location, since the maximu m load normally takes place only during a short time period, the load cycles of the consumers are different, and therefore, the best substation location changes for each hourly load scenario, and will displace continuously in the plane describing a closed trajectory. On the other hand, the deterministic calculation of an equivalent load center may find obstacles for the final selection of the substation site due to a number of constraints that are present in a real situation. In this article, a probabilistic methodology is presented to calculate the perimeter of the area where the load center has maximum likelihood to be found, taking into account in the planning process the hourly load changes or load cycle. With this perimeter calculated, the final decision of locating the substation site should be made within the calculated area, considering the availability of the land lots, the market prices, and several other factors.

A probabilistic methodology is presented to assist the planning engineers in the selection of the distribution substation location. The methodology considers the hourly load changes and calculates an estimate probability distribution in order to determine a recommended perimeter for the selection of the substation location associated to the high-probability area for a given probability level. Three different probability distribution types were successfully tested on a realistic case, and results are presented showing the potential of the proposed approach. The resultant perimeter represents valuable information for the for the location of the distribution substation, where the availability and the cost of the land lots as well as other relevant constraints that may be present in the selection of the substation site must be considered, especially in urban areas.

S. Subramaniam et al.[1] have developed a new knowledge-based algorithm using multi-temporal spectral information available in four bands of Advanced Wide Field Sensor (AWiFS) on board ResourceSat-1 (with spatial resolution of $56 \mathrm{~m}$ ) namely Green (G), Red (R), Near Infrared (NIR) and Short Wave Infrared (SWIR) for inventorying and monitoring of various types water bodies. The algorithm has been applied for the data obtained from other space-borne sensors with similar spectral bands such as Landsat ETM, IRS LISS III and ASTER and found to be working satisfactorily. Results were validated by comparing the results reported from other popular methods. The study provides a quick method for generation of spatio-temporal water body's information. 
This will be helpful for development of Water bodies Information System (WIS) on national/global scales. This is almost similar to our project; here the spatial data is analyzed and clustered to find the water bodies in a given area. Here they have used the clustering algorithms to not only to cluster the data, but also to eliminate noises like clouds and so on.

In literature many clustering algorithms are available like DBRS+ [5], COD_CLARANS[10], AutoClust+ [11], DBCluC[12], DBRS_O[13] etc. Xin Wang, Camilo Rostoker, and Howard J. Hamilton [5] propose a new spatial clustering method, called DBRS +, which aims to cluster spatial data in the presence of both obstacles and facilitators. It can handle datasets with intersected obstacles and facilitators. Without preprocessing, DBRS+ processes constraints during clustering. It can find clusters with arbitrary shapes and varying densities. DBRS + has been empirically evaluated using synthetic and real data sets and its performance has been compared to DBRS, AUTOCLUST+ [11], and DBCLuC [12]. This paper talks about a new density based clustering algorithm, which clusters the data in the presence of obstacles (such as, mountains, rivers, highways and so on). It also provides a new feature called facilitators that are used to connect data objects across obstacles. These improve the efficiency of and provides a better clustered data output.

The review of literature in the field has spotlighted some limitations in the existing work that are as follows:

1. The existing systems use polygons for modeling obstacles. Polygons are highly intensive in computation and space.

2. The spatial proximity is not very clear due to the usage of Polygons for its identification.

3. The modeling of obstacles is weak when polygons are considered.

4. Most of the existing systems use density based clustering algorith ms and graph based clustering in the presence of obstacles has not been explored much.

\section{Proposed Work}

With the proposed system an entrepreneur can locate potential places for his business. The detailed stepwise approach is as follows:

1. Accept the city name, type of business and the constraints applied on the business for which the locations are to be identified.

2. Identify the city center on the globe, and hence identify various locations in and around the city.

3. Extraction of various attributes - spatial and nonspatial, which describe the location.
4. Pruning the unwanted and invalid data points (locations) from the dataset as per the constraints and other basic conditions.

5. Constructing incremental Delaunay Triangles for all valid data points for capturing spatial proximity.

6. Identifying various obstacles in the vicinity of the city and categorizing them.

7. Based on the category some obstacles are modeled with Bezier Curves.

8. Delaunay Triangles and Bezier Curves are overlapped and the intersecting edges in Delaunay Triangles are eliminated. Other techniques for elimination of data points not following the constraints are also implemented.

9. The data points are identified as clusters having similar characteristics.

10. Obtained clusters are mapped to a Graphical Interface for easy identification of locations.

11. A detailed report is also generated.

The code snippet for data collection is as follows:

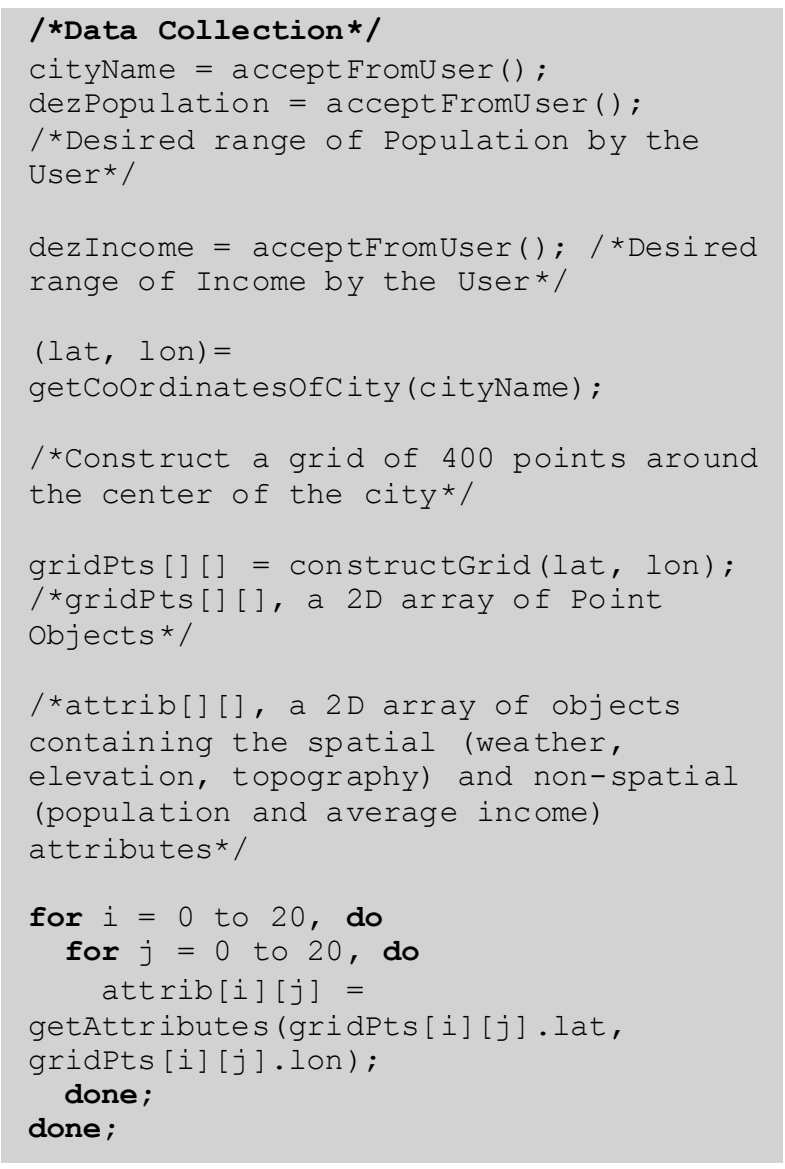

The essential information about a business enterprise is accepted through a web interface. The information includes the city name, type of business and some constraints on setting up the business, like population, average income per person in the location and so on. 
Based on the city name specified, the location of the central point of the city is identified. The locations within the radius of 19 to $20 \mathrm{~km}$ from the center of the city are determined. For all the determined locations, the spatial information like elevation from sea level and weather details, and non-spatial information like population and average income per person for each location are extracted from Google XML pages.

Thus, the collected information for each location is pre-processed to remove unwanted and invalid data points. Following is the code for data pre-processing:

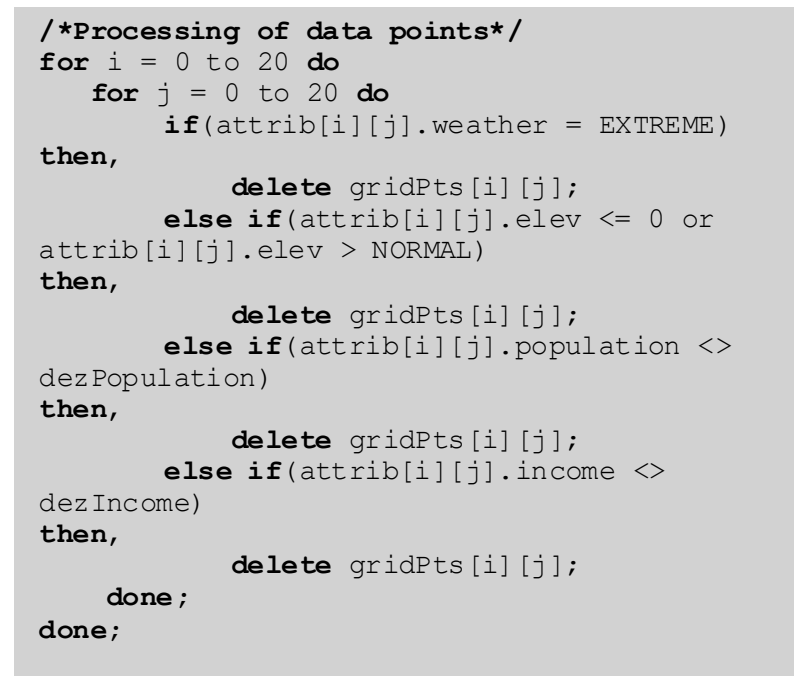

The extracted information may contain redundant entries and some data points may violate the constraints specified. The pseudo code to remove these data points is as shown below.

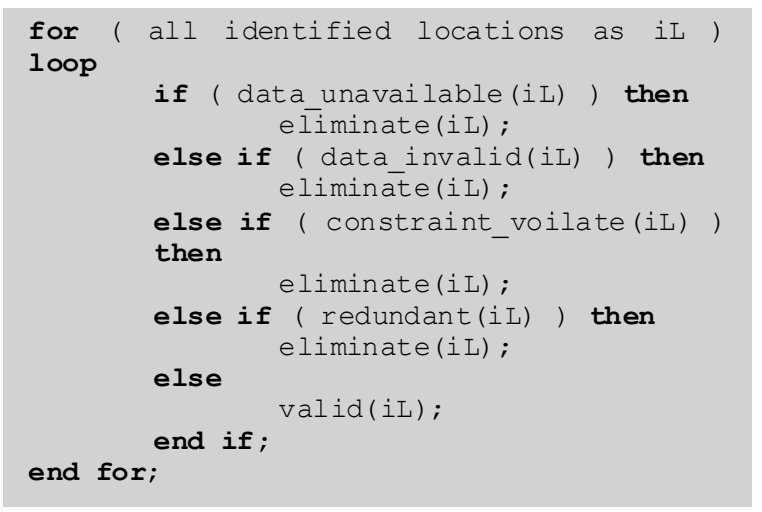

The unwanted, unnamed, redundant and constraint violated data are removed. Some data points may lay out of the specified city (termed as unwanted points) and some data points may fall under the same locality of the city (termed as redundant points). Some data points may not satisfy some basic conditions or may not satisfy the constraints specified by the user. Such points are also eliminated.

The obtained valid data points are subjected to "Incremental Delaunay Triangulation" [6, 7]. Delaunay triangles are used to find the spatial proximity between data points (locations) efficiently. Initially, an edge is drawn between the closest two points and the third point of the triangle should lie on the circumcircle, circumscribing these three points. Similarly, the triangles are constructed for all the data points in an incremental fashion.

Obstacles are data points that are not suitable for establishing business enterprises as they do not satisfy certain constraints or fail to meet some basic conditions. The characteristics of an obstacle point are different from the surrounding valid data points. At this phase, the data points can be obstacle points based on the irregularity in spatial attributes describing them. Obstacle points can either be a single data point (lake or pond or a mountain) or a sequence of data points( like river, highway, mountain range or border of a state or country) that can be modeled as a curve. Thus, these obstacles should be identified and categorized appropriately. The pseudo code for identify ing obstacles is given below:

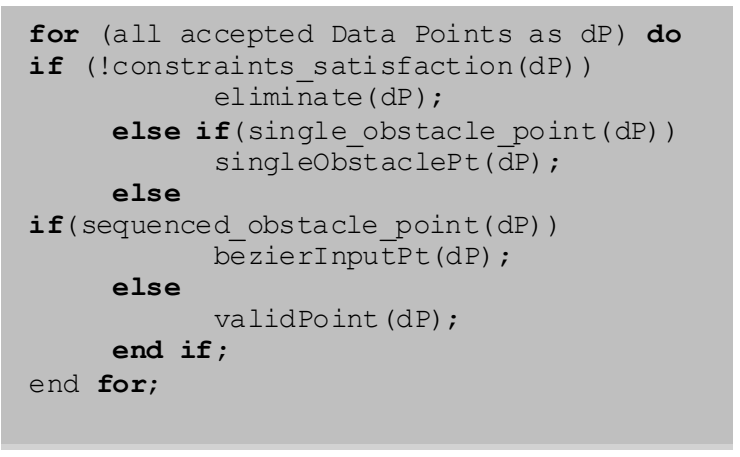

Based on the categories identified in the previous stage, the obstacles that are a sequence of data points are modeled using Bezier Curves [8,9]. The Bezier Curve approximates the shape of the obstacle points. This is shown in the following figure 5.

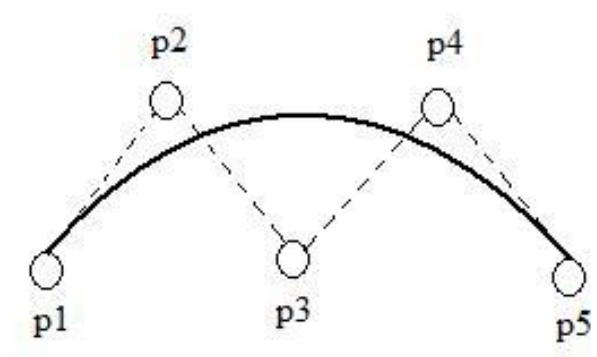

Fig. 5: Bezier curves are used to model the shape of an obstacle using the obstacle points

The points $\mathrm{p} 1, \mathrm{p} 2, \mathrm{p} 3, \mathrm{p} 4$ and $\mathrm{p} 5$ are treated as the obstacle data points in the vicinity of clusters. Points $\mathrm{p} 1$ and $\mathrm{p} 5$ are termed as the end points and the remaining are the control points.

Delaunay Triangles are used to capture the spatial proximity between data points and Bezier curves are used to approximate the shape of the obstacle points. After the construction of these two structures they are 
overlapped such that the edges of the Delaunay triangles that are intersected by the Bezier curves are rejected. The characteristics of the overlapped edges fall under the category of obstacles. This divides a big group of points into smaller sets of them, forming smaller Delaunay Triangles. Some of the data points that do not satisfy the given constraints are also eliminated by checking if any of those points overlap with the considered set of Delaunay Triangle's edges or points.

Figures 6,7 and 8 show the complete process of eliminating intersecting edges and thus clustering of data points.

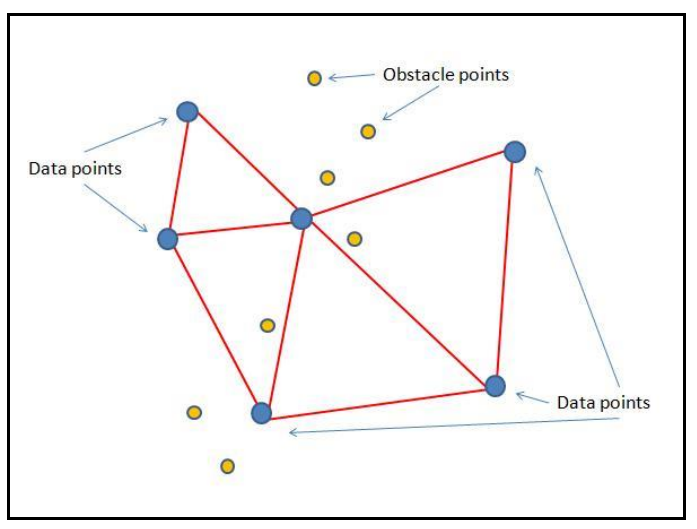

Fig. 6: Delaunay Triangles before merging

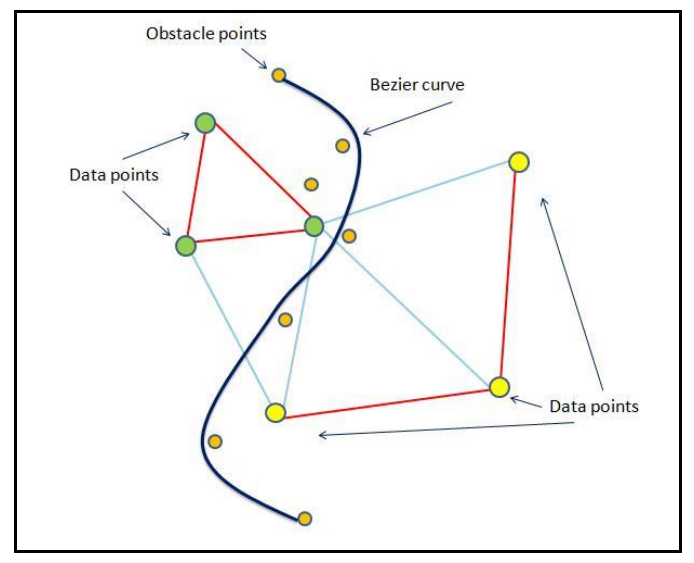

Fig. 7: The complete process of clustering

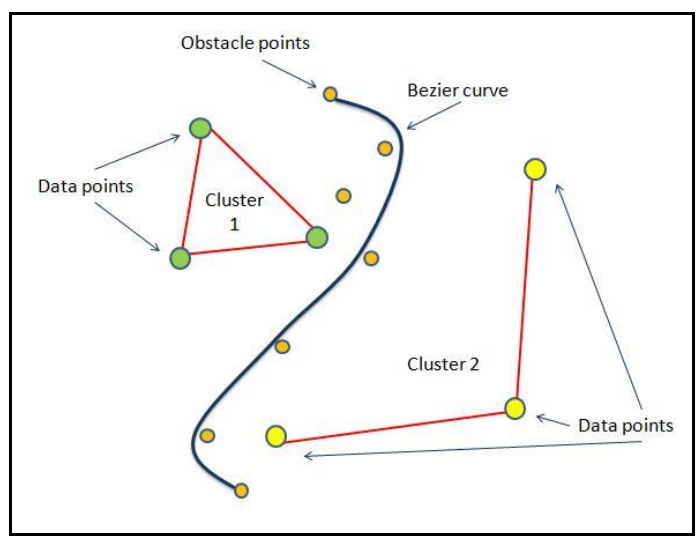

Fig. 8: After eliminating obstacles
The data points that are similar to each other based on certain characteristics and different from rest of the points are grouped together in one cluster. The following code snippet illustrates the complete process of clustering:

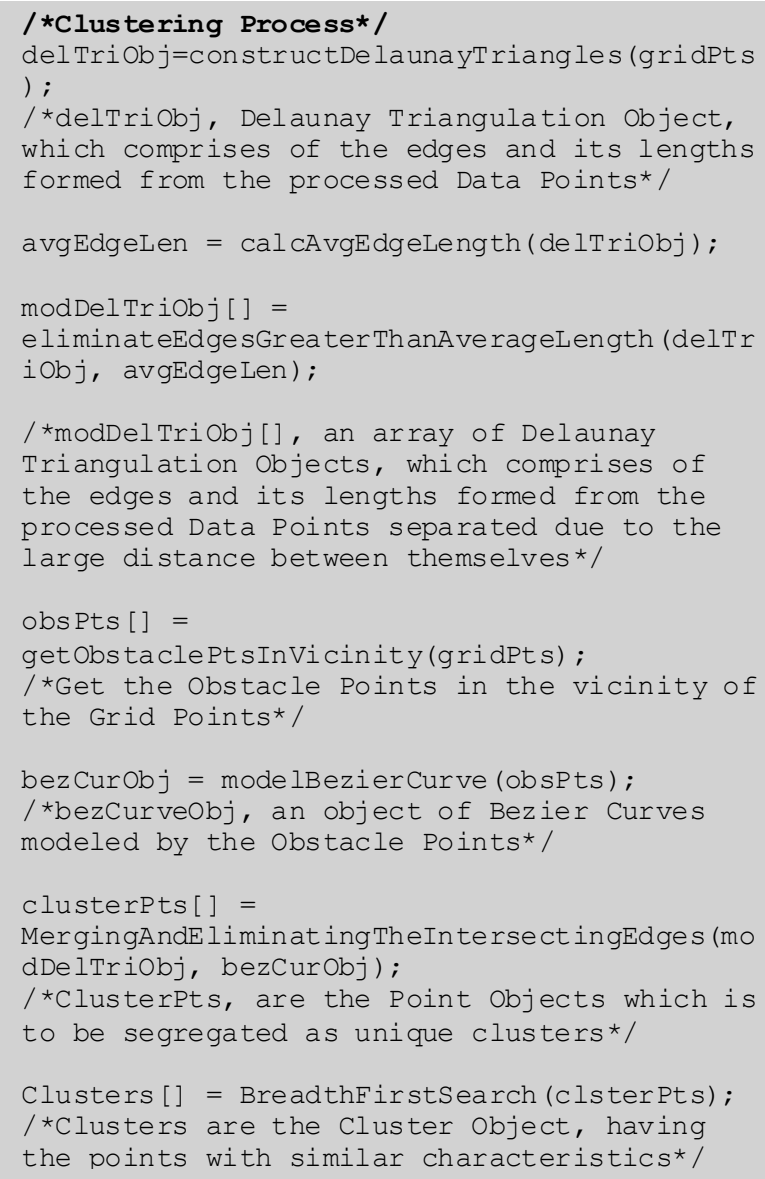

The small groups of Delaunay Triangles obtained from the previous stage justify these. The BFS algorithm is implemented in order to identify the connected sub-graphs in a completely disconnected graph. Each cluster is represented using a different color.

The clusters of data points obtained are mapped on to Google maps using Map Marker APIs in JavaScript V3. This facilitates easy identification of clusters and location details.

Each cluster is given a Unique Cluster Number (UCN). A report is also generated which comprises all the details of the clusters and its data points. This is highly useful for the user to understand various locations obtained as clusters of data points.

\section{Experiment and Analysis}

The following are the experimental results from the test run of the application being developed. A cluster is identified by a color in the graphical representation using Google Maps. The colors are repeated due to limited number of colors provided by Google. 
Hence, similar color points far from each other belong to different clusters. To avoid ambiguity, a detailed PDF report is generated by the application. The experiments are explained in three cases:

\subsection{Case - Mumbai}

The vector format of the data points is accepted in the application by implementing the algorithm. This is shown in figure 9.

\begin{tabular}{|c|c|c|c|}
\hline \multicolumn{4}{|r|}{ Mumbai } \\
\hline $\begin{array}{c}\text { Serial } \\
\text { Number }\end{array}$ & Latitude & Longitude & Address \\
\hline 1 & 18.9759837 & 72.8076559 & Navroji Gamadia Cross Rd, Breach Candy, Mumbai, Maharashtra, India \\
\hline 2 & 18.9759837 & 72.8176559 & Bhanjibhai Rathod Ln, Arya Nagar, Mahalakshmi, Mumbai, Maharashtra, India \\
\hline 3 & 18.9759837 & 72.8276559 & Maulana Azad Rd N, Agripada, Mumbai, Maharashtra, India \\
\hline 4 & 18.9759837 & 72.8376559 & St Savta Marg, Mustafa Bazar, Byculla, Mumbai, Maharashtra, India \\
\hline 5 & 18.9759837 & 72.8476559 & St Savta Marg, Darukhana, Byculla, Mumbai, Maharashtra, India \\
\hline 6 & 18.9859837 & 72.8176559 & Lala Lajpathrai Marg, Mahalakshmi, Mumbai, Maharashtra, India \\
\hline 7 & 18.9859837 & 72.8276559 & Jr Boricha Marg, Adarsh Nagar, Lower Parel, Mumbai, Maharashtra, India \\
\hline 8 & 18.9859837 & 72.8376559 & Tukaram Bhikaji Kadam Marg, Ghodapdeo, Byculla, Mumbai, Maharashtra, India \\
\hline 9 & 18.9859837 & 72.8476559 & Third Ave, Sewree East, Sewri, Mumbai, Maharashtra, India \\
\hline 10 & 18.9859837 & 72.8576559 & Haji Bandar Rd, Sewri, Mumbai, Maharashtra, India \\
\hline 11 & 18.9959837 & 72.8176559 & Dr E Moses Marg, Bheem Nagar, Mahalakshmi, Mumbai, Maharashtra, India \\
\hline 12 & 18.9959837 & 72.8276559 & Sitaram Jadhav Marg, Lower Parel, Mumbai, Maharashtra, India \\
\hline
\end{tabular}

Fig. 9: Accepted Locations of Mumbai in Vector format

Mumbai is a location in India that is surrounded by water bodies as shown in figure 10 . This is considered in order to show that the clustering algorithm does not include any huge water bodies like rivers and oceans. Figure 11 shows the various clusters being formed after the completion of the whole process.

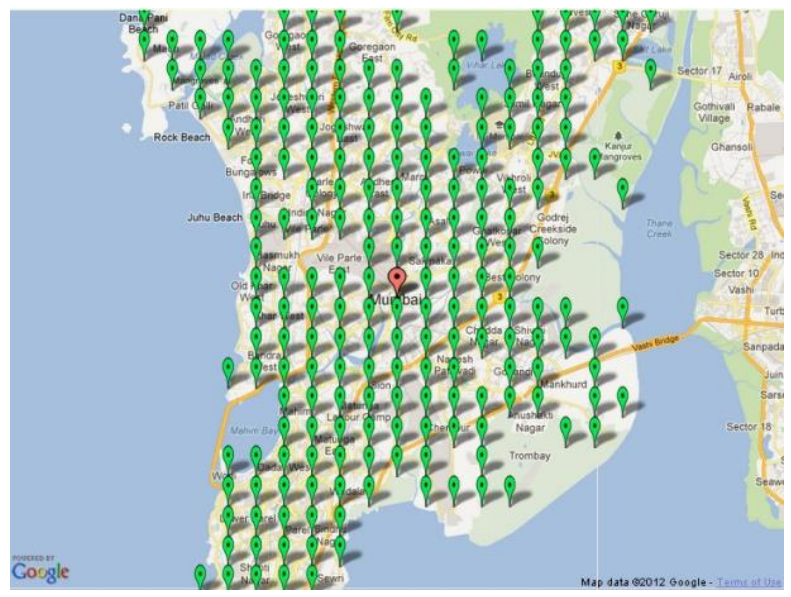

Fig. 10: Graphical representation of the accepted locations of Mumbai

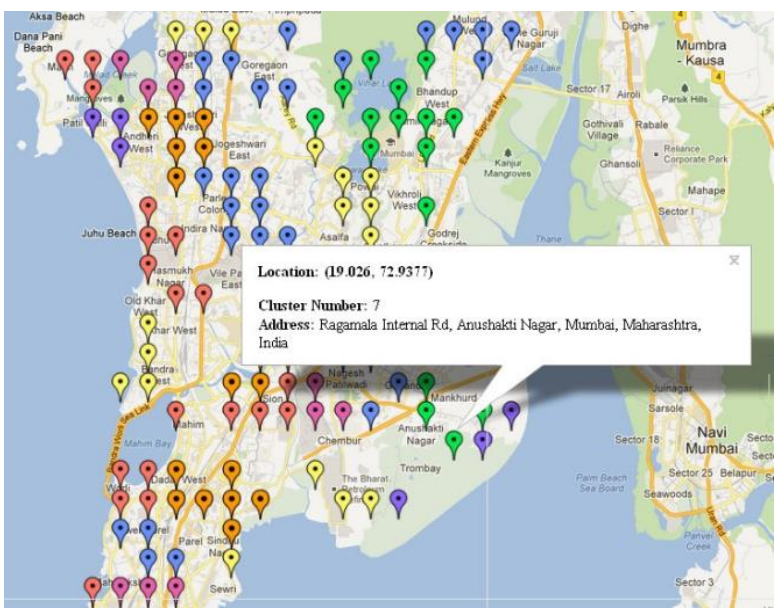

Fig. 11: The output with identified clusters for Mumbai

\subsection{Case - Patna}

Patna is a location in India that has the river Ganges running through it. This is considered in order to show that the clustering algorithm identifies the rivers as an obstacle and models it using the Bezier Curves. Figure 12 shows the vector format of the data points accepted by the algorithm. 
Implementing Delaunay Triangles and Bezier Curves to

\begin{tabular}{|c|c|c|c|}
\hline \multicolumn{3}{|c|}{ Patna } \\
\hline $\begin{array}{c}\text { Serial } \\
\text { Number }\end{array}$ & Latitude & Longitude & Address \\
\hline 1 & 25.51046 & 85.041667 & State Highway 78, Neva, Bihar, India \\
\hline 2 & 25.51046 & 85.051667 & State Highway 78, Neva, Bihar, India \\
\hline 3 & 25.51046 & 85.061667 & State Highway 78, Neva, Bihar, India \\
\hline 4 & 25.51046 & 85.071667 & National Highway 22, Bihar, India \\
\hline 5 & 25.51046 & 85.081667 & National Highway 22, Bihar, India \\
\hline 6 & 25.51046 & 85.091667 & National Highway 22, Bihar, India \\
\hline 7 & 25.51046 & 85.101667 & National Highway 22, Bihar, India \\
\hline 8 & 25.51046 & 85.111667 & Punpun Rd, Bihar, India \\
\hline 9 & 25.51046 & 85.121667 & Punpun Rd, Bihar, India \\
\hline 10 & 25.51046 & 85.131667 & Punpun Rd, Bihar, India \\
\hline 11 & 25.51046 & 85.141667 & Punpun Rd, Bihar, India \\
\hline 12 & 25.51046 & 85.151667 & Punpun Rd, Bihar, India \\
\hline \hline
\end{tabular}

Fig. 12: Accepted Locations of Patna in Vector format

Figure 13 represents all the locations accepted by the algorithm and Figure 14 show the clusters being obtained.

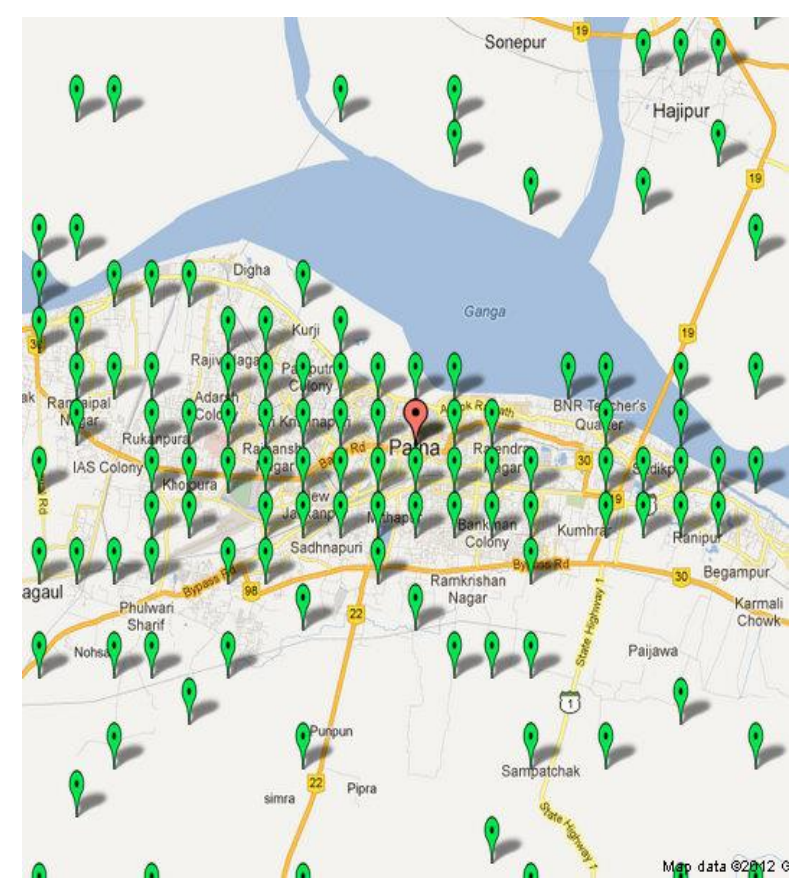

Fig. 13: Graphical representation of the accepted locations of Patna

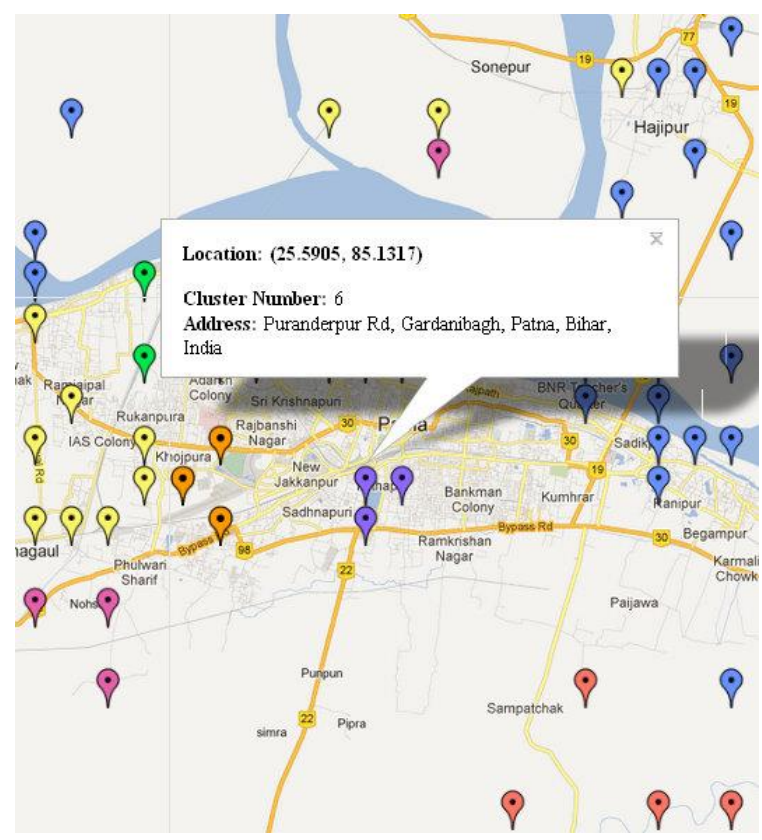

Fig. 14: The output with identified clusters for Patna

\subsection{Case - Mysuru}

Mysuru is a location in India that has the landmasses surrounding it. This is considered to demonstrate the algorith ms action in complete land masses. Here in case 3 also, figure 15 shows the vector format of the data points extracted, figure 16 represents these data points on Google map and figure 17 represents the clusters so obtained after eliminating all unnecessary and unwanted data points.

\begin{tabular}{|c|c|c|c|}
\hline \multicolumn{4}{|r|}{ Myssuru } \\
\hline $\begin{array}{c}\text { Serial } \\
\text { Number }\end{array}$ & Latitude & Longitude & Address \\
\hline 1 & 12.1958104 & 76.5393805 & Mananthavadi Rd, Karmataka, India \\
\hline 2 & 12.1958104 & 76.5493805 & Mananthavadi Rd, Karnataka, India \\
\hline 3 & 12.1958104 & 76.5593805 & Manantharadi Rd, Karmataka, India \\
\hline 4 & 12.1958104 & 76.5693805 & Manantharadi Rd, Karnataka, India \\
\hline 5 & 12.1958104 & 76.5793805 & Mananthavadi Rd, Karnataka, India \\
\hline 6 & 12.1958104 & 76.5893805 & Mananthavadi Rd, Karnataka, India \\
\hline 7 & 12.1958104 & 76.5993805 & Mananthavadi Rd, Karnataka, India \\
\hline 8 & 12.1958104 & 76.6093805 & Thalore Bus Station, Karnataka, India \\
\hline 9 & 12.1958104 & 76.6193805 & Mananthavadi Rd, Karnataka, India \\
\hline 10 & 12.1958104 & 76.6293805 & Mananthavadi Rd, Karnataka, India \\
\hline 11 & 12.1958104 & 76.6393805 & Mananthavadi Rd, Karnataka, India \\
\hline 12 & 12.1958104 & 76.6493805 & Kozhikode-Mysore-Kollegal Hwy, Kamataka, India \\
\hline
\end{tabular}

Fig. 15: Accepted Locations of Mysuru in Vector format 


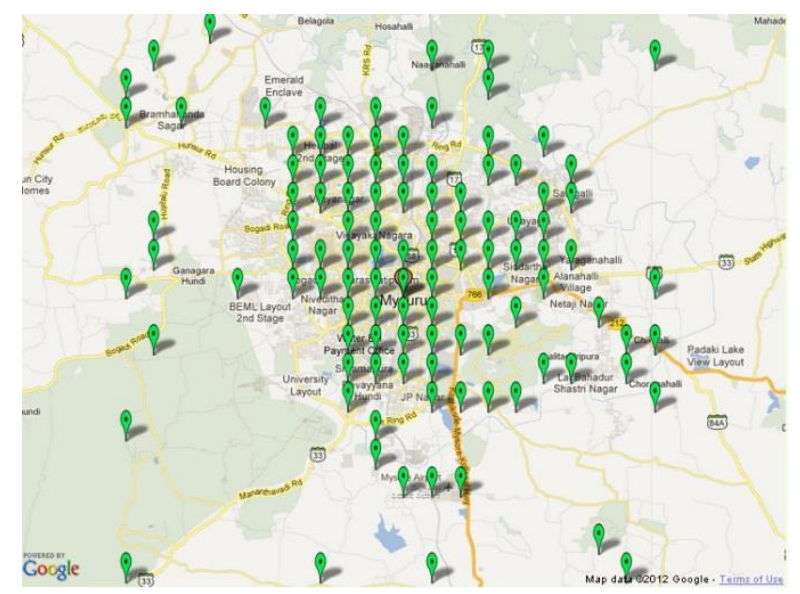

Fig. 16: Graphical representation of the accepted locations of Mysuru

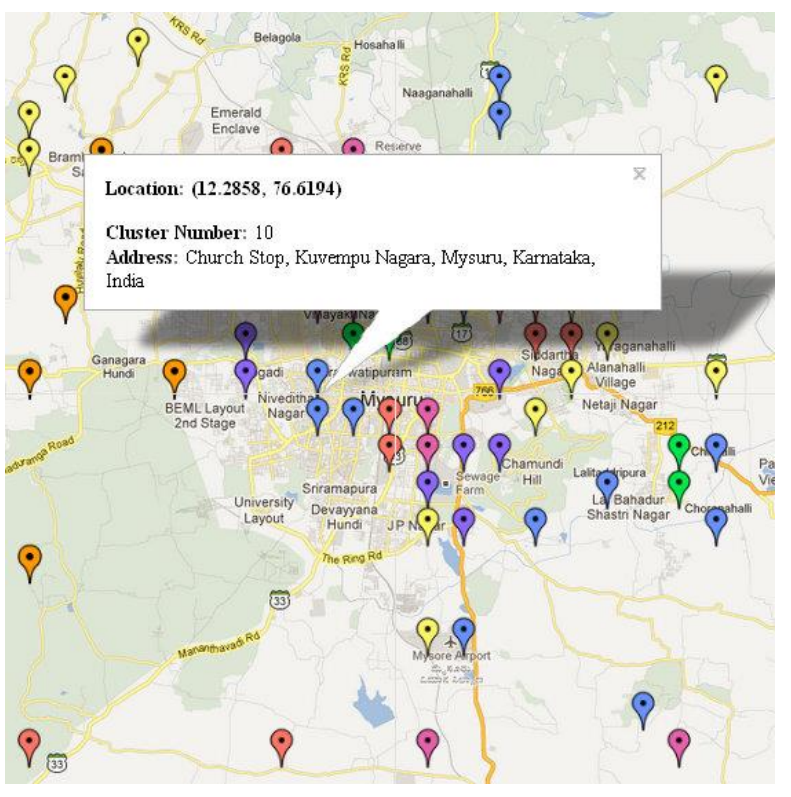

Fig. 17: The output with identified clusters for Mysuru

The application has been tested for various other places and the results so obtained are very encouraging.

\section{Conclusion and Future Directions}

This application is most useful to entrepreneurs to setup enterprises in most suitable locations in the cities known or unknown to them. It uses data from Google databases obtained in the form of XML and JSON pages by querying it with appropriate URLs. Locations all over the world are described using spatial attributes obtained from Google pages. These definitions are further improved by considering some non-spatial attributes that decides or affects the various factors of a business enterprise.

This application implements a spatial graph based clustering algorith $m$ that involves two major geometric structures: Delaunay Triangles and Bezier Curves. Delaunay Triangles are those computational structures that are used to capture spatial proximity and thus help in forming the desired clusters. Bezier curves are used to approximate the obstacles present in the vicinity. Well-defined clusters are obtained by overlapping both Delaunay triangles and Bezier Curves. These clusters are then identified on Google Maps using unique colors and a detailed PDF report is also being generated.

Future Enhancements can be as follows:

1. Considering the Facilitators in order to nullify some of the obstacles and hence improve the quality of clusters.

2. Considering more number of non-spatial attributes in order to refine the clusters obtained as per the constraints specified by the end user.

3. Data extraction from the Google databases can be improved in terms of Time complexity.

4. Considering more number of data points in and around the city under consideration. Currently it is around four hundred points. The number of points can be made dynamic based on the size of the city.

\section{References}

[1] S. Subramaniam, A. V. Suresh Babu, and Partha Sarathi Roy, "Automated Water Spread Mapping Using ResourceSat-1 AWiFS Data for Water Bodies Information System" in IEEE J. of selected topics in applied earth observations and remote sensing, Vol. 4, No. 1, March 2011.

[2] Xiang Guo, Dong Yan, Jianrong Fan, Wanze Zhu Mai-He Li, "Evaluating the ecological suitability for Olive tree in Sichuan Province using GIS and comprehensive fuzzy method: Methodological development and application" in Computing in Science \& Engineering, Sept. 2009.

[3] H. M.Khodr, Jorge A. Melián, Adolfo J. Quiroz, Danie la C.Picado, José María Yusta, and Alberto J. Urdaneta,"A Probabilistic Methodology for Distribution Substation Location" in IEEE Transactions on Power Systems, Volume. 18, Number. 1, February 2003

[4] Zhi-Qiang Liu, "Contextual Fuzzy Cognitive Map for Decision Support in Geographic Information Systems" in IEEE Transactions on Fuzzy Systems, Volume. 7, No. 5, Oct 1999.

[5] Xin Wang, Camilo Rostoker, and Howard J. Hamilton "Density-Based Spatial Clustering in the Presence of Obstacles and Facilitators" In Proc. of PKDD 2004.

[6] The voronoi website: www.voronoi.com

[7] Sibson, Robin, The Dirichlet Tessellation as an aid in Data Analysis. In Scandavian J. of Statistics, 1980. 
[8] Pu S 'Managing Freform Curves \& Surfaces in a spatial DBMS. Master Thesis, July 2005.

[9] www.tdplines.com/resources/class notes/BezierCurve.html

[10] Tung A.K.H., Hou J., and Han J.: Spatial Clustering in the Presence of Obstacles. In Proc. of Intl. Conf. on Data Engineering (ICDE'01), Heidelberg, Germany,2001, pp. 359-367.

[11] Estivill-Castro V. and. Lee I.J.: AUTOCLUST+: Automatic Clustering of Point-Data Sets in the Presence of Obstacles. In Proc. of the Intl. Workshop on Temporal, Spatial and SpatialTemporal Data Mining, Lyon, France, 2000, pp. 133-146.

[12] Zaïane O. R., and Lee C. H.: Clustering Spatial Data When Facing Physical Constraints. In Proc. of the IEEE International Conf. on Data Mining, MaebashiCity, Japan, 2002, pp.737-740

[13] Wang X. and Hamilton H.J.: Density-based spatial clustering in the presence of obstacles. In Proc. of $17^{\text {th }}$ Intl. Florida Artificila Intelligence Research Society Conference (FLAIRS 2004), pp.312-317, Miami.

[14] Han, J. and Kamber, M. Data Mining: Concepts and Techniques, Morgan Kaufmann Publishers, 2006.

[15] Atsuyuki O., Barry B., Kokichi S, Sungnok C: Spatial Tessellations: Concepts \& Applications of Voronoi Diagrams, $2^{\text {nd }} E d ., 2000$.

\section{Authors' Profiles}

Tejas Pattabhi has completed his Bachelor of Engineering in Information Science from PESIT Bangalore South Campus in July 2012. Currently, he is working as Junior System Administrator in Societe Generale-Global Solution Center. His research interests include Spatial Data Mining, Geographical Information Systems, Internet technologies.

Arti Arya has completed BSc(Mathematics Hons) in 1994 and MSc(Mathematics) in 1996 from Delhi University. She has completed her Doctorate of Philosophy in Computer Science Engineering from Faculty of Technology and Engineering from Maharishi Dayanand University, Rohtak, in 2008. Her areas of interest include spatial data mining, knowledge based systems, text mining, unstructured data management, applied numerical methods and biostatistics. She is a life member of CSI and member IEEE. She is on the reviewer board of many reputed International Journals. She is currently serving as Professor and Head of the Department (MCA), PES Institute of Technology, Bangalore South Campus. She has more than twelve years of teaching and six years of research experience.
Pradyumna N has completed his Bachelor of Engineering in Information Science from PESIT Bangalore South Campus in July 2012. He is currently working as Software Engineer in HCL-BOPT ETS, Bangalore. His research interests include Knowledge based systems, spatial clustering techniques, unstructured database management.

Swati Singh has completed her Bachelor of Engineering in Information Science from PESIT Bangalore South Campus in July 2012. Her interest areas are unsupervised learning techniques and Geographical Information Systems.

Sukanya Dhansingh has completed her Bachelor of Engineering in Information Science from PESIT Bangalore South Campus in July 2012. Her areas of interest include spatial data mining \& spatial clustering techniques.

How to cite this paper: Tejas Pattabhi, Arti Arya, Prady umna N, Swati Singh, Sukanya D,"Implementing Delaunay Triangles and Bezier Curves to Identify Suitable Business Locations in the Presence of Obstacles", International Journal of Information Technology and Computer Science(IJITCS), vol.5, no.3, pp.29-39, 2013.DOI: 10.5815/ijitcs.2013.03.04 